\title{
Seizures: a rare presentation of Sheehan's syndrome
}

\author{
M S A Cooray ${ }^{1}$, Uditha Bulugahapitiya ${ }^{2}$, D D Ranasinghe ${ }^{3}$ \\ Sri Lanka Journal of Diabetes, Endocrinology and Metabolism 2013; 3: 88-90
}

\begin{abstract}
Sheehan's syndrome is a well-known cause of panhypopituitarism following ischemic damage to the pituitary gland or stalk during peripartum period. Degree of hypopituitarism in Sheehan's syndrome can vary and due to the slow evolution, the diagnosis can be delayed. Here we report a case presented to us with hyponatremia, 2 years after her complicated delivery, which highlights the importance of recognizing hyponatremia as a presentation of hypopituitarism in Sheehan's syndrome.
\end{abstract}

Key words: hyponatremia, Sheehan syndrome

\section{Introduction}

Sheehan's syndrome, first described by Sheehan in 1937 (1), is a well-known cause of panhypopituitarism following ischemic damage to the pituitary gland or hypothalamic-pituitary stalk during peripartum period (2) and this is usually due to intrapartum or postpartum haemorrhage with severe hypotension or hemorrhagic shock (Table 1). Vasospasm, thrombosis and vascular compression of the hypophyseal arteries have also been described as possible causes (3). Rarely, it may occur even after a normal uncomplicated delivery without significant amount of bleeding. Although the pathogenesis of Sheehan's syndrome is not completely clear, widespread ischemia is considered to be the main reason for the impairment of anterior pituitary function. Onset of the disease, degree of hypopituitarism and disease presentation can vary. Here we describe a patient with Sheehan's syndrome

Table 1. Criteria for diagnosis of Sheehan's syndrome

1. Typical obstetric history of postpartum vaginal bleeding.

2. Severe hypotension or shock for which blood transfusions or fluid replacement was needed.

3. Failure of postpartum lactation.

4. Failure to resume regular menstruation after delivery.

5. Varying degree of anterior pituitary failure (partial or panhypopituitarism).

6. Empty sella on CT or MRI scan of the pituitary. who presented 2 years after the possible ischaemic episode with recurrent convulsions due to severe hyponatraemia.

\section{Case Report}

A 39 year old woman has got admitted to a medical casualty unit with generalized tonic clonic convulsions, which had been preceded by altered behaviour, headache and vomiting. Following the seizures, the patient had been drowsy with impaired level of consciousness. While in the ward, she has undergone extensive investigations that included cerebro-spinal fluid (CSF) analysis and CT imaging of the brain, which did not reveal any abnormality. She was treated empirically with intravenous antibiotics, antiviral treatment (Acyclovir) and intravenous steroids for suspected meningitis after which she recovered. During this admission, she was also found to have hyponatremia, which was attributed to syndrome of inappropriate $\mathrm{ADH}$ secretion (SIADH) and managed conservatively. She dramatically improved with the course of antibiotics and steroids and on discharge was asymptomatic and well. She was discharged on antiepileptic drugs but following discharge her health lasted for only a few days after which she became confused with severe vomiting and then went on to develop seizures. She was re-admitted to the medical casualty unit several days later with recurrent seizures. The initial response to intravenous anti-epileptic drugs was poor and she was found to be hypotensive and needed fluid resuscitation. Recurrent central nervous system infection was suspected and she was again treated with intravenous steroids and antibiotics. However, the CSF analysis was normal and blood and CSF cultures did not yield any organisms. Her biochemical investigations revealed severe hyponatraemia ranging from 116 to 124

${ }^{1}$ Senior Registrar in Endocrinology, ${ }^{2}$ Consultant Endocrinologist, Colombo South Teaching Hospital, ${ }^{3}$ Consultant Radiologist, Colombo South Teaching Hospital, Kalubowila, Sri Lanka. 
$\mathrm{mmol} / \mathrm{L}$ with marginal hyperkalaemia. She also had haematological evidence of normocytic normochromic anaemia with a haemogobin of $8.8 \mathrm{~g} / \mathrm{dl}$. Her symptoms resolved with the initiation of intravenous steroids and fluid resuscitation and her serum sodium got normalised.

Her past history revealed a pregnancy complicated by pre-eclampsia, 2 years prior to onset of convulsions, for which she underwent an emergency caesarean section. Following delivery, she had developed postpartum haemorrhage with shock, for which she required multiple blood transfusions. Subsequently, she had lactation failure and experienced asthenia and fatigue with loss of appetite and weight. These symptoms were attributed to anaemia and were treated with haematinics. However, she didn't have any improvement with the symptoms and could not tolerate even a minor illness. Although she had regained menstruation 6 months after the delivery, her sex desire has been less and she was not fertile despite unprotected sexual exposure. She denied a history of polyuria and polydipsia.

Clinical examination revealed sparse pubic and axillary hair with normal adult type external genitalia. Hormonal assays revealed a 9 am cortisol of $2.69 \mu \mathrm{g} / \mathrm{dl}$ (5-25) and post ACTH stimulation cortisol level of $7.9 \mu \mathrm{g} /$ dl. Her free T4 level was $0.77 \mathrm{ng} / \mathrm{dl}$ with a TSH of $1.36 \mu \mathrm{IU} /$ $\mathrm{mL}$. Her LH, FSH and estradiol levels were $2.08 \mathrm{u} / \mathrm{L}, 2.38$ $\mathrm{u} / \mathrm{L}$ and $59 \mathrm{pg} / \mathrm{mL}$ respectively. Based on these findings, she was diagnosed as having panhypopituitarism. Pituitary imaging with a MRI scan of the pituitary revealed atrophied anterior pituitary, which was compatible with a diagnosis of pituitary infarction (Figure 1) (4).

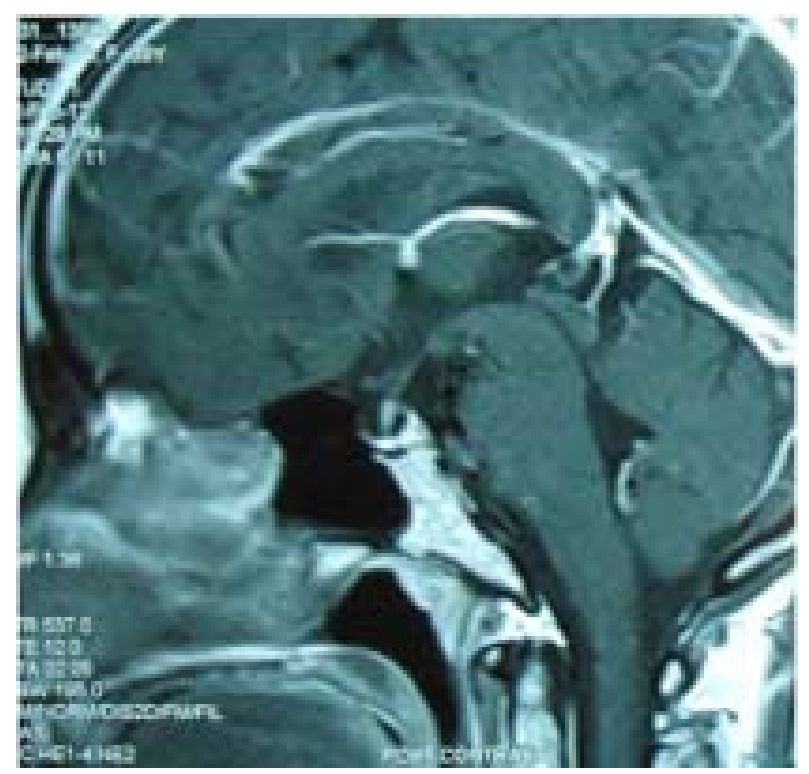

Figure 1. Gadolinium enhanced T1-weighted MR pituitary saggital image show central low signal intensity with a thin rim of enhancing tissue along the sellar floor. CSF is noted in the sella ("empty sella").
She was started with pituitary replacement therapy with hydrocortisone and levothyroxine sodium. Her clinical condition, serum sodium and haemoglobin levels improved and reached normal range with hormone replacement.

\section{Discussion}

Hypothyroidism, adrenal insufficiency, hypogonadism, growth hormone deficiency, hypoprolactinemia, and different sodium and water disturbances are welldescribed clinical features of Sheehan's syndrome (3). Enlargement of pituitary gland, small sella size, disseminated intravascular coagulation and autoimmunity have been suggested to play a role in the development of Sheehan's syndrome in women who suffer from severe postpartum haemorrhage. Although a small percentage of patients with Sheehan's syndrome may present with severe hypopituitarism immediately after delivery, majority have mild disease and go undiagnosed and untreated for years. It may result in partial or panhypopituitarism and the great majority of the patients have empty sella on CT or MRI scan of the pituitary (4).

Lack of lactation and failure of menstrual resumption after a delivery are the most important clues to the diagnosis of Sheehan's syndrome (5). Although our patient had lactation failure, she continued to have menstrual cycles until presentation, which may have probably delayed the diagnosis of hypopitutarism. Gonadotrophic function may be preserved in an occasional patient. There have been several reports of patients with Sheehan's syndrome who maintained regular menstrual cycles and even became pregnant spontaneously (7-9). Thus, the absence of amenorrhea or the presence of postpartum lactation, does not rule out the diagnosis.

The occurrence of sodium and water disturbances associated with Sheehan's syndrome depends on the degree of pituitary damage, time of onset since the initial pituitary insult, and concurrent medical conditions (6). Hyponatremia is not a disease, but a manifestation of a variety of disorders and it may be the only manifestation of hypopituitarism or hypothyroidism. The diagnosis of hypopituitarism in hyponatraemic patients is often overlooked and it is a common electrolytic disorder, occurring in $33 \%$ to $69 \%$ of all cases with Sheehan's syndrome (10).

Several mechanisms are responsible for hyponatremia in patients with Sheehan's syndrome. Hypothyroidism and glucocorticoid deficiency by decreasing free water clearance independent of vasopressin cause hyponatremia. Syndrome of inappropriate antidiuretic hormone secretion (SIADH) and volume depletion can also lead to hyponatremia. Volume depletion, cortisol deficiency and hypothyroidism are the causative factors for hypo- 
natraemia in our patient and this was easily corrected with salt replacement with hydrocortisone and thyroxine treatment (10-13).

Panhypopituitarism is often accompanied by normocytic normochromic anaemia, which is usually mild and seldom below $9 \mathrm{~g} / \mathrm{dL}$. This is due to cortisol deficiency, hypothyroidism and hypogonadism. It has shown that glucocorticoids stimulate erythropoiesis and thyroid hormone stimulates erythropoietin production and proliferation of erythroid progenitor cells (2). Our patient's haemoglobin level improved with adequate cortisol and thyroxine replacement.

Although Sheehan's syndrome is uncommon as a result of improved obstetric care, it should be a consideration in any woman who has a history of a postpartum hemorrhage and who reports signs or symptoms of pituitary deficiency. Replacement of deficient hormones is important not only to correct endocrine abnormalities, but also to reduce mortality due to hypopituitarism (5). In patients who have secondary hypothyroidism and hypocortisolism, glucocorticoids should be replaced before the replacement of thyroid hormone. Gonadotropin deficiency and hypogonadism should be treated with hormone replacement therapy.

This case highlights the importance of identifying hyponatraemia as a presentation of hypopituitarism and the possibility of Sheehan's syndrome when presented after childbirth especially with vague and varied clinical features, even if all pituitary axes seem to be unaffected.

\section{References}

1. Sheehan HL. Postpartum necrosis of the anterior pituitary. J Pathol Bact 1937; 45: 189-214.

2. Pham PC, Pham PA, Pham PT. Sodium and water disturbances in patients with Sheehan's syndrome. Am J Kidney Dis. 2001; 38(3): E14.
3. Anfuso S, Patrelli TS, Soncini E, Chiodera P, et al. A case report of Sheehan's syndrome with acute onset, hyponatremia and severe anemia. Acta Bomed 2009; 80: 73-6.

4. Kelestimur F. Sheehan's syndrome. Pituitary 2003; 6(4): 181-8.

5. Sert M, Tetiker T, Kirim S, Kocak M. Clinical report of 28 patients with Sheehan's syndrome. Endocr J. 2003; 50(3): 297-301.

6. Shivaprasad C. Sheehan's syndrome: Newer advances. Indian J Endocrinol Metab 2011; 15(Suppl 3): 203-7.

7. Moreira AC, Zanini Maciel LM, Foss MC, Tabosa Veríssimo JM, Iazigi N. Gonadotropin secretory capacity in a patient with Sheehan's syndrome with successful pregnancies. Fertil Steril 1984; 42: 303-5.

8. Westbrock DA, Srivastava LS, Knowles HC. Jr Preservation of normal menstrual cycles in a patient with Sheehan's syndrome. South Med J. 1983; 76: 1065-7.

9. Zargar AH, Masood SR, Laway BA. Pregnancy in Sheehan's syndrome: A report of three cases. J Assoc Physicians India 1998; 46: 476-8.

10. Kurtulmus N, Yarman S. Hyponatremia as the presenting manifestation of Sheehan's syndrome in elderly patients. Aging Clin Exp Res. 2006; 18(6): 536-9.

11. Shoji M, Kimura T, Ota K. Cortical laminar necrosis and central pontine myelinolysis in a patient with Sheehan syndrome and severe hyponatremia. Intern Med 1996; 35 : 427-31.

12. Putterman C, Almog Y, Caraco Y, Gross DJ, Ben-Chetrit E. Inappropriate secretion of antidiuretic hormone in Sheehan's syndrome: A rare cause of postpartum hyponatremia. Am J Obstet Gynecol. 1991; 165: 1330-3.

13. Singhania P, Singh S, Banerjee R, Singhania B, Banerjee I, Maitra S. Hyponatremia - A rare and emergency presentation of Sheehan's Syndrome. Pak J Med Sci. 2010; 26: 713-5. 